\begin{tabular}{|l|l|c|c|}
\hline Eiszeitalter $u$. Gegenwart & 35 & $\begin{array}{c}205-210 \\
3 \mathrm{Abb} .\end{array}$ & Hannover 1985 \\
\hline
\end{tabular}

\title{
Kleinformen von Strukturböden in den Hochlagen des Bayerischen Waldes
}

\author{
Ulrich HaUner *)
}

Pedogensis, miniature forms, sorted polygons, parent materials, upper zones.

Bavarian Forest (Großes Falkenstein-Gebiet), Bayern,

TK 25: Nr. 7044

$\mathrm{Kurz}$ fas s u n g: Erstmals werden rezente Erdknospen und Texturböden aus den Hochlagen eines deutschen Mittelgebirges beschrieben. Untersuchungen der Form, des Substrats und der Frosteffekte belegen ihre Entstehung als Strukturböden. Im Vordergrund der Untersuchung stehen die heutigen Bedingungen für die Weiterentwicklung und Erhaltung der Form. Dem feuchtigkeitsbedingten Aufquellen des thixotropen Substrats im Sommerhalbjahr und der Bildung von Eiskörnchen an Eistagen kommt eine große morphogenetische Bedeutung für die Kleinformen zu.

[Miniature forms of sorted polygons in the upper zones of "Bayerischer Wald"]

A b s t r a ct: This is the first description of recently developed Erdknospen (soil buds) and Texturböden (structured soils) from the upper zones of a medium hight mountainous region in Germany. Investigations of their form, their substratum and the frost effects give evidence of their genesis as sorted polygons. The most important aspect is that of the present conditions for the further development and the preservation of these miniature forms. The swelling up of the thixotropic substratum due to humidity in summer, and the crystallization of ice granules on frosty days is of great morphogenetic importance.

\section{Einleitung}

Dem frdl. Hinweis von Dr. G. PROPACH/München auf Kleinpolygone am Großen Falkenstein folgend, suchte der Verf. in den vergangenen Jahren Hochlagengebiete des Inneren Bayerischen Waldes ab und konnte außer dem in einem Abri gelegenen Strukturbodenfeld nahe dem Gipfel des Großen Falkensteins (1300 m ü. N. N., S/NWExposition, 33 Erdknospen, 25 Polygone in einem Texturboden) drei kleinere Erdknospenfelder an der Südflanke des Falkensteins (1285 m ü. N. N., NNE-Exposition), östlich des Wanderwegs von der Rachelkapelle zum Rachelgipfel im Nationalpark Bayerischer Wald (1265 m ü. N. N., SW/W-Exposition) und oberhalb des Wald-

*) Anschrift des Autors: Dr. U. Hauner, Floriansmühlstr. 13, 8000 München 45. 
schmidthauses im Nationalpark (1380 m ü. N. N., SW/W-Exposition) finden und untersuchen. Begehungen in der Gipfelregion des Arbers, sowie entlang des LusenGrenzkamms erbrachten trotz der häufigen Leitform Felsburg keine weiteren Funde.

Den vier Vorkommen ist neben der Höhenstufe (Gipfelregion des Mittelgebirges mit hohen Nebelniederschlägen: 200 bis $300 \mathrm{~m}$ unterhalb der hypothetischen Waldgrenze) die mikroklimatisch bedeutsame Schutzlage unterhalb von Frostkliffs und in Klüften von Felsburgen, die Vegetationsfreiheit (zugleich Voraussetzung als auch Folge bodendynamischer Prozesse im Strukturbodenfeld) einer maximal $2^{\circ}$ geneigten Bildungsfläche, das Fehlen oberflächiger Abspülung, Feuchtigkeitszufuhr allein durch Nebel und geringe Mengen von Sickerwasser, aber auch ein dichter Felsuntergrund als Wasserstauer gemein.

Bei den Erdknospen handelt es sich um locker gefügte, weichplastische Feinerdehöcker mit einem Durchmesser von 5 bis $20 \mathrm{~cm}$, die 1 bis $4 \mathrm{~cm}$ über der Umgebung aufgewölbt sind. Größere Einzelformen ab $10 \mathrm{~cm}$ Durchmesser haben sich über mehr als $6 \mathrm{~cm}$ mächtigem Substrat gebildet und sind stets von aussortiertem Grobmaterial umkränzt.

Bei Detritustiefen von weniger als $4 \mathrm{~cm}$ treten keine Erdknospen mehr auf, statt dessen finden wir einen Texturboden (im Gegensatz zu Zellenböden ohne Steinchen im polygonalen Rißnetz), wenn zwei Bedingungen erfüllt sind: Erstens darf die Korngrößenverteilung des Substrats nur ein Spektrum von tonig bis feinsandig (mit einem Maximum von mehr als $50 \%$ in der Fraktion von 2 bis $36 \mu$ ) aufweisen, zweitens muß das Substrat in durchschnittlich feuchten Sommern fast bis zur Übersättigung durchfeuchtet sein. Am Großen Falkenstein konnte beobachtet werden, daß sich in den Sommerhalbjahren 1982 und 1983 niemals das polygonale Muster verlor, in der trockenkalten Hochwinterphase aber alle Risse geschlossen waren. Offensichtlich wird gegen Ende der Frostwechselperiode die Fläche des Texturbodens noch vor dem benachbarten Erdknospenfeld stark durchfeuchtet, so daß Frostrisse auftreten. Dabei erreicht in diesen Tagen das Porengefüge des Substrats - das thixotrope Eigenschaften zeigt seinen Ausgleichszustand in den Grenzen der Polygone, so daß das Netz erhalten bleibt. Welche Rolle spielen Frosteffekte aber bei der Bildung der Erdknospen?

\section{Untersuchung der bodendynamischen Prozesse im Erdknospenfeld auf dem Großen Falkenstein}

Idealen Bedingungen entsprechend liegt das schon 1963 beobachtete Strukturbodenfeld unweit des Falkensteingipfels auf einem meterlangen, ebenen Felssockel, der durch eine 1,5 m hohe Steinstufe vom Gras- und Farnunterbewuchs des Bergfichtenwaldes deutlich abgesetzt ist. Es wird von einem vier meter weit vorspringenden Abri aus Cordieritgneis vor Regen und Schnee geschützt.

Messungen vom Oktober 1982 bis Mai 1983 an Erdknospen des Strukturbodenfeldes zeigten, daß trotz der großen Anzahl von 63 Frostwechseltagen (Der Deutsche Wetterdienst ermittelte in der nur $80 \mathrm{~m}$ westlich gelegenen Klimastation im Mittel der letzten 30 Jahre 81 Frostwechseltage) der Skelettanteil des Hügelsubstrats kaum in Bewegung war. Zu Versuchszwecken waren markstückgroße Plastikplättchen 1 und 


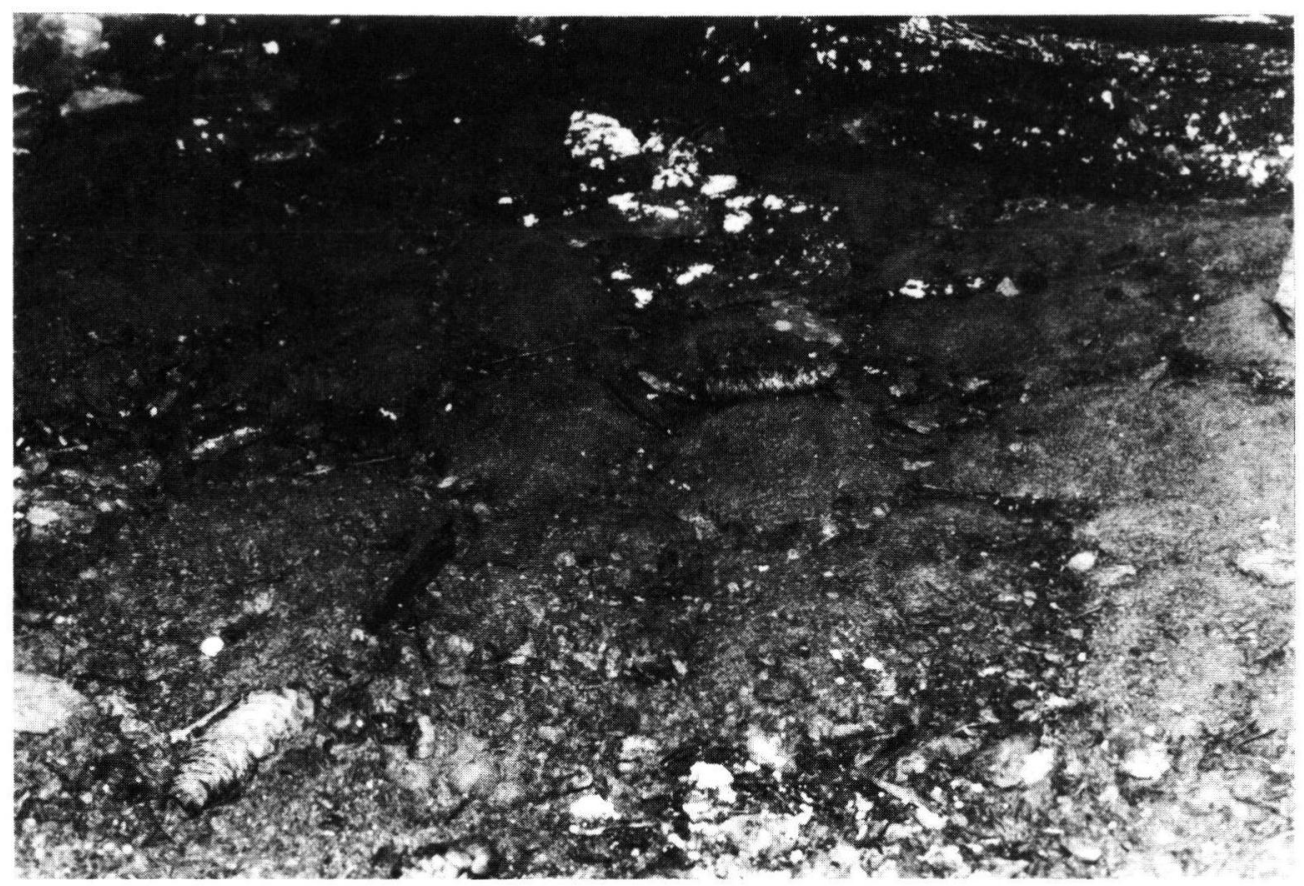

Abb. 1: Rezentes Erdknospenfeld am Großen Falkenstein (1300 m ü. N. N.) (Bildbreite $70 \mathrm{~cm})$.

$2 \mathrm{~cm}$ tief unter die flachkonvexe Erdknospenoberfläche eingeschoben worden. Diese veränderten mit Ausnahme eines Plättchens, das sich im Übergangsbereich ErdknospeSteinrahmen befand und am Platz deutlich nach außen schräg gestellt wurde, ihre Lage nicht. Dieser Befund überrascht, selbst wenn man in Rechnung stellt, daß die geringe winterliche Tagesamplitude der Temperatur sowie die nur $1 \frac{1 / 2}{2}$ stündige direkte Sonneneinstrahlung, welche spontaner eine Bodentemperaturänderung bewirkt als die Änderung der Lufttemperatur, nicht leicht einen Bodenfrostwechsel erzeugen kann.

Ein Grund für die nicht erfolgte Bewegung im Hügel selbst ist sicherlich auch im geringen winterlichen Durchfeuchtungsgrad der oberen Substratschicht zu suchen: Der Bodenwassergehalt lag am Ende der Frostwechselperiode 1982/83 deutlich unter $10 \%$, was wohl darauf zurückzuführen ist, daß analog der Gefriertrocknung bald nach dem Einsetzen der Frostwechselperiode durch mehrmaliges Gefrieren und Auftauen Eis der obersten Bodenschicht infolge nackter Oberfläche verdunstet. Das entstehende Feuchtedefizit kann durch die ansonsten nicht zu unterschätzenden Nebelniederschläge (Nebelstundenzahl von Oktober bis März $46 \% \pm 9$ im 30jährigen Mittel) nicht kompensiert werden. Bei überdurchschnittlichem winterlichen Feuchteangebot und günstiger Windrichtung darf jedoch angenommen werden, daß eine frostbedingre Bodensortierung stattfinden kann. Das Plastikplättchen im etwas tiefer gelegenen und damit in der Frostwechselperiode etwas feuchteren Randbereich der Erdknospen im durchschnittlich trockenen Winter 1982/83 wurde ja bereits bewegt.

Die Analyse der Korngrößenverteilung ergab, daß das Material der Erdknospen sehr gut sortiert ist. Die Summenkurven des Granulogramms des Hügelzentrums liegen 


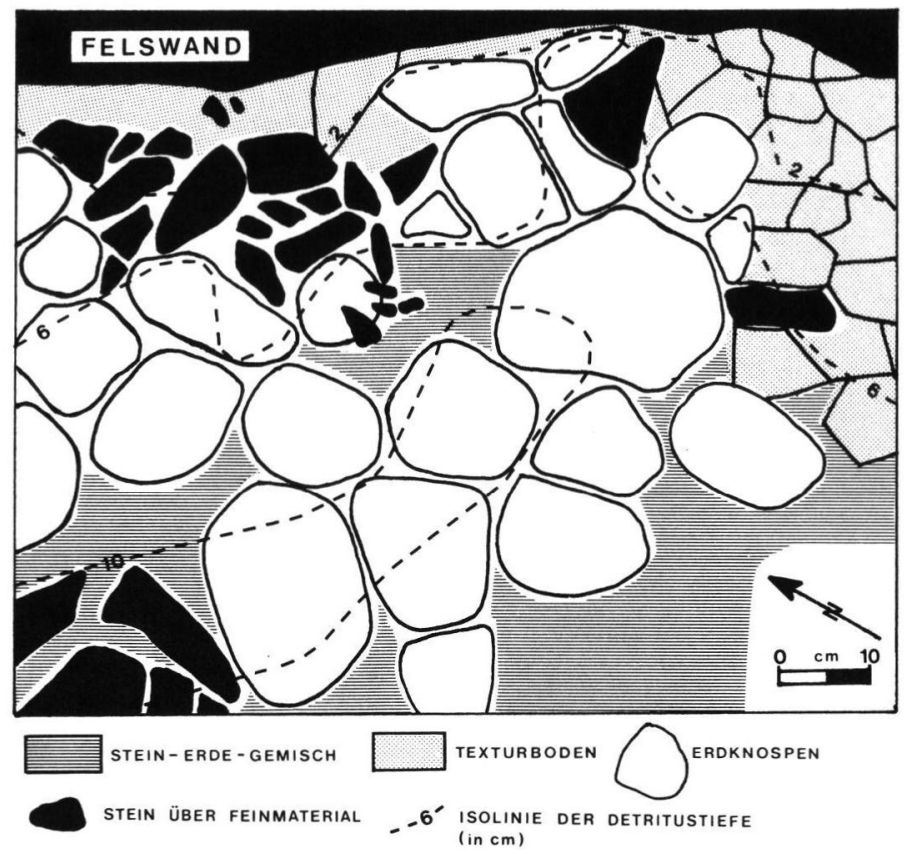

Abb. 2: Zentraler Bereich des Strukturbodenfeldes am Großen Falkenstein (1300 m).

ideal in der von GraF (1973) angegebenen Bandbreite solifluidaler Böden in den Alpen. Auffällig ist ein Schluffanteil von ca. 45 Gewichts\%. Hingegen liegt das Korngrößenmaximum im Substrat am Hügelrand mit $40 \%$ in der Fraktion Mittelkies. Damit ist eine weitgehende Materialsortierung erreicht.

Als Agens für den Feldversuch mit den Plastikplättchen hätte prinzipiell Kammeis in Betracht kommen können, es wurde jedoch nicht beobachtet. Unter den auf einige Erdknospen gelegten Gesteinsscherben bildeten sich im Laufe der Frostwechselperiode zwar zahlreiche halbmillimetergroße Eiskörnchen, die als Ausgangspunkt für die Kristallisation von Kammeis ideal gewesen wären: Die für diesen Prozeß aber notwendige Feuchtigkeit war nicht vorhanden.

Allerdings konnten im Substrat der Erdknospen Eiskörnchen bis zu $1,5 \mathrm{~mm}$ Durchmesser beobachtet werden, die grundsätzlich jenen von FURRER (1954) und FREUND (1972: $98 \mathrm{ff}$.) beschriebenen entsprechen. Sicherlich stehen sie auch in unserem Untersuchungsgebiet in einem genetischen Zusammenhang mit kugeligen Hohlräumen der frostfreien Periode, worauf noch eingegangen wird. Die Eiskörnchen bildeten sich an Eistagen des Winters 1982/83 in einer 1 bis $2 \mathrm{~cm}$ mächtigen Schicht unterhalb der ausgetrockneten Hügeloberfläche. Im Liegenden zeigte sich eine Wechselfolge komprimierter Eis-Substrat-Lamellen, die an Taber-Eislamellen erinnern.

Im Frühjahr 1983 konnte beobachtet werden, daß dieser liegende Schichtverband nach mehreren frostfreien Tagen allmählich seine Struktur verlor und begann, ein porenreicheres Gefüge aufzubauen. Zwei Wochen nach dem Ende der Frostwechselperiode 1982/83 zeigte sich dann im ganzen Bodenprofil ein homogenes, porenreiches 


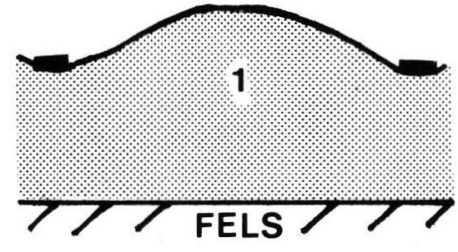

a) Sommerhalbjahr

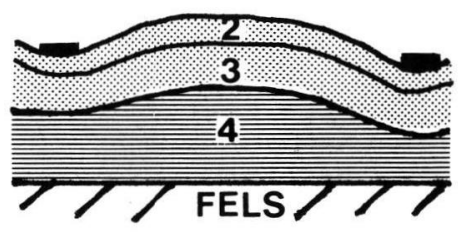

b) Eistag
Abb. 3: Querprofil durch zwei Idealformen einer Erdknospe im Sommer und Winter
1 feuchtes Substrat mit luftgefüllten Poren
2 trockenes Substrat mit luftgefüllten Poren
3 gefrorenes Substrat mit Eiskörnern
4 horizontale Eis-Substrat-Lamellen

Gefüge (Abb. 3a), die Bodenstruktur hatte sich regeneriert. Die Bodenporen werden dann erst wieder zu Beginn der nächsten Frostwechselperiode durch den zunehmenden Kristallisationsdruck im unteren Bereich des Substrats zerstört. In den mittleren und oberen Lagen der Erdknospen bilden sich in den kugeligen, wassergefüllten Poren Eiskörnchen als Auffüllungspseudomorphosen. Ein Frostdruck von morphologischer Bedeutung entsteht aber nur dann, wenn alle Poren ganz mit Wasser gefüllt sind. In der Frostwechselperiode 1982/83 war dies aber nicht der Fall.

Die Entstehung der blasenartigen Hohlräume liegt in der Struktur des schluffreichen Substrats selbst begründet: Es zeigt bei der üblichen sommerlichen weichplastischen Konsistenz die Eigenschaft der Thixotropie. Im trockenen Mai 1983 konnte sie erst in Detritustiefen von 0,7 bis $1,0 \mathrm{~cm}$ beobachtet werden. Damals wurde die natürliche Anordnung der Bodenteilchen durch Spatenstiche verletzt und sofort ging das Substrat in eine zähflüssige Konsistenz über, weil das nun entstandene porenärmere Gefüge das adsorbierte Wasser teils auspreßte. Das Substrat war beim nächsten Exkursionstermin, vierzehn Tage später, weichplastisch, ein Hügel war jedoch noch nicht entstanden. Im Sommer 1983 ergab sich jedoch eine Volumenzunahme um $15 \%$ durch Wasseraufnahme. SCHEFFER \& SCHACHTSCHABEL (1973: 203) betonen, daß sich bei thixotropen Böden die Teilchen nach der Beanspruchung wieder ordnen und erneut die alten Positionen eines Minimums an freier Energie und eines Maximums an Anziehung einnehmen. Dieses Bestreben könnte nach der trockenheitsbedingten Winterstarre 1982/83 der Erdknospenoberfläche Ursache für die beobachtete Volumenvergrößerung der Gesamtform im Frühsommer sein. Dieses feuchtigkeitsbedingte Ausblühen der Winterform verhindert den Ansatz von Flechten und Moosen und bestimmt die räumliche Verteilung aktiver und inaktiver Flächen des Erdknospenfeldes in der Vegetationsperiode. In diesem Sinne ist durch die große Trockenheit im Sommer 1983 das untere Drittel der in Abb. 2 wiedergegebenen Fläche im Oktober noch inaktiv gewesen. Die klimatischen Verhältnisse im Sommer spielen also eine nicht unerhebliche Rolle beim Fortbestehen des Strukturbodenfeldes. 


\section{Schriftenverzeichnis}

FreUND, R. (1972): Vergleichende Betrachtung von Kleinformen der Solifluktion im Raume Mittelbünden (Schweiz), auf West-Spitzbergen und am Kilimandjaro. — 111 S.; Zürich (Juris).

FURRER, G. (1954): Solifluktionsformen im Schweizerischen Nationalpark. — Diss. Univ. Zürich, Liestal. - 75 S.; Zürich.

GrAF, K. (1973): Vergleichende Betrachtungen zur Solifluktion in verschiedenen Breitenlagen. - Z. Geomorph. N. F., Suppl. Bd. 16: 104-154; Berlin · Stuttgart.

HAUNER, U. (1980): Untersuchungen zur klimagesteuerten tertiären und quartären Morphogenese des Inneren Bayerischen Waldes (Rachel-Lusen) unter besonderer Berücksichtigung pleistozän kaltzeitlicher Formen und Ablagerungen. - Regensburger Geogr. Schr., 14: 198 S.; Regensburg.

Scheffer, F. \& Schachtschabel, P. (1973): Lehrbuch der Bodenkunde. - 448 S.; Stuttgart.

Manuskript eingegangen am 29. 7. 1984 